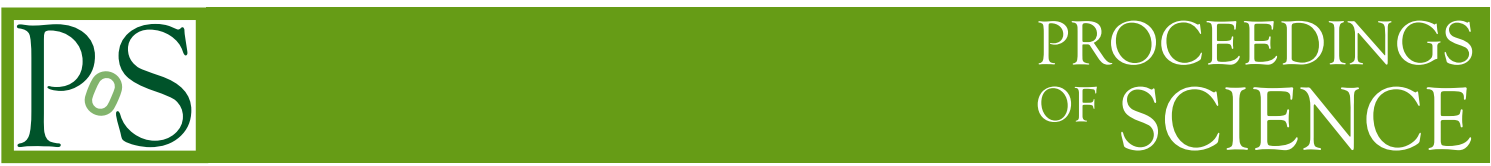

\title{
Recent highlights and plans of the AWAKE experiment
}

\author{
Giovanni Zevi Della Porta* on behalf of the AWAKE Collaboration \\ CERN, \\ Geneva, Switzerland \\ E-mail: gzevi@cern.ch
}

The Advanced Wakefield Experiment (AWAKE) is an accelerator R\&D experiment at CERN using, for the first time, a high-energy proton bunch to drive wakefields in plasma and accelerating electrons to the $\mathrm{GeV}$ energy scale. The principle of the AWAKE experiment is described. We show experimental results of the seeded self-modulation process of the long $400 \mathrm{GeV}$ SPS proton bunch, transforming the bunch into a train of micro-bunches and driving resonantly the wakefields in the $10 \mathrm{~m}$ long $\mathrm{Rb}$ plasma. We also show that externally-injected electrons can be accelerated by these wakefields to several GeV. The next steps of the AWAKE experimental program are shown. Possible first applications to high-energy physics experiments, where the scheme takes advantage of the large energy stored in the proton bunch to reach very high energy gain in a single plasma, are described.

40th International Conference on High Energy physics - ICHEP2020

July 28 - August 6, 2020

Prague, Czech Republic (virtual meeting)

${ }^{*}$ Speaker 


\section{Run 1 (2016-2018): setup and experimental results}

The first data taking period of the Advanced Wakefield Experiment (AWAKE) [1] successfully achieved two important milestones: the longitudinal self-modulation of a proton beam in a plasma, and the acceleration of externally-injected electrons.

As shown in Figure 1, a long proton beam is self-modulated as it interacts with the Rb plasma, forming micro-bunches at the characteristic frequency of the plasma. While the frequency of the modulation can be controlled by adjusting the plasma density, the phase of the modulation is controlled by 'seeding' the self-modulation process with a large transverse wakefield, thus creating a fully deterministic and repeatable process [2-4]. Electron acceleration results are summarized in Figure 2: a $19 \mathrm{MeV}$ electron beam is injected into the self-modulated proton beam, and the longitudinal wakefields driven by the proton micro-bunches provide acceleration to a subset of the electrons. Energies of up to $2 \mathrm{GeV}$ are reached at the end of the $10 \mathrm{~m}$ long plasma, with a maximum accelerated charge of approximately $100 \mathrm{pC}$, corresponding to a fifth of the charge of the initial electron beam. As expected from theory and simulations, the acceleration is affected by the plasma density and by the density gradient along the path of the beams [5].
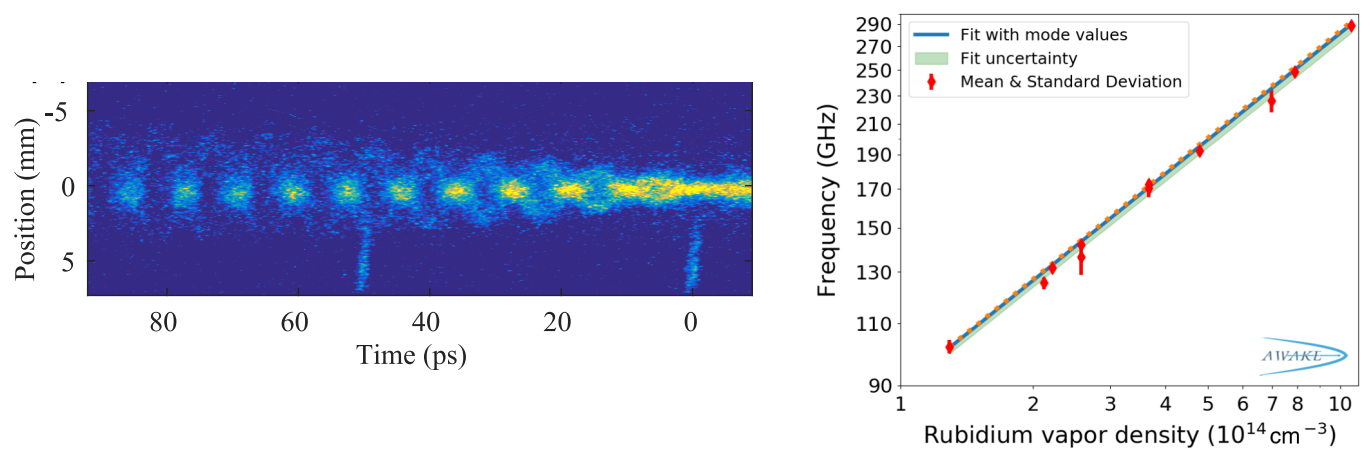

Figure 1: (Left) Streak camera image of self-modulated proton beam. (Right) Measured frequency of self-modulation as a function of plasma density [2-4].
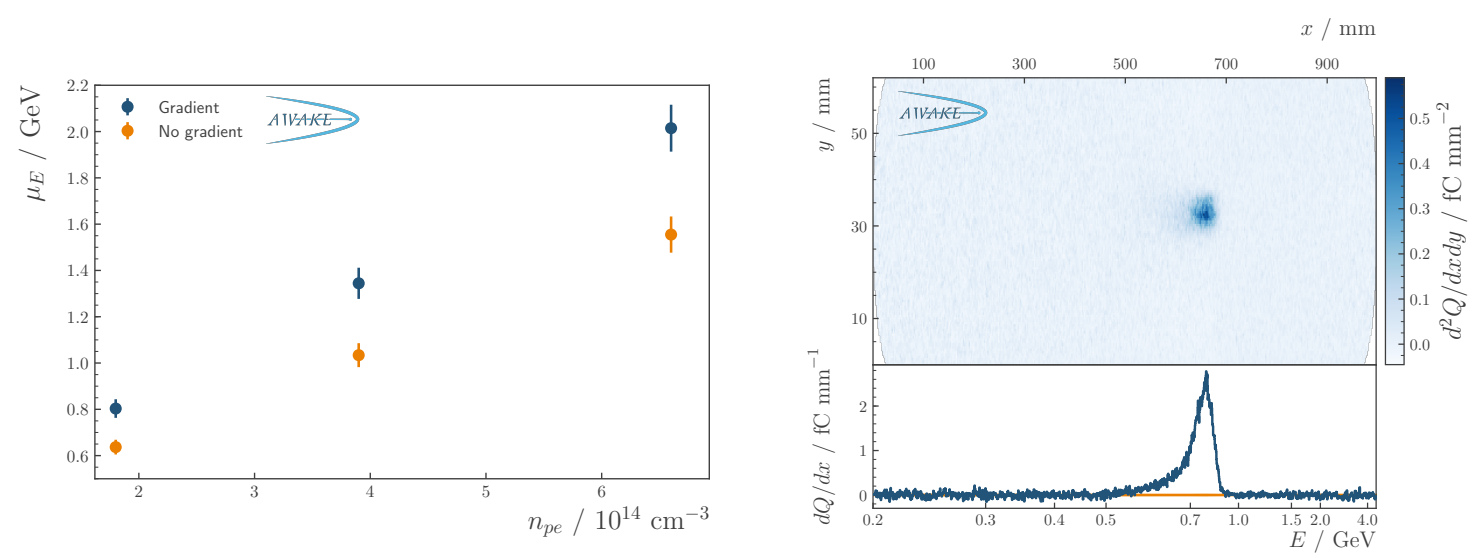

Figure 2: (Left) Measured energy of accelerated electrons as a function of plasma density for different density gradients. (Right) Signal of accelerated electrons on the spectrometer scintillator for a single event [5]. 


\section{Run 2: goals and milestones}

Building upon the success of Run 1, an ambitious set of goals and milestones has been defined for the AWAKE Run 2 project [6]. The overall aim of Run 2 will be to demonstrate the scalability of the AWAKE scheme for future particle physics applications, discussed in the next section. The goals of Run 2 are: (i) accelerate an electron beam to high energy (with a gradient of $0.5-1 \mathrm{GV} / \mathrm{m}$ ), (ii) preserve electron beam quality and (iii) demonstrate a scalable plasma source technology which can support up to and beyond $100 \mathrm{~m}$ of plasma.

The first milestone, in Run 2a, will be to demonstrate that the proton bunch self-modulation can be seeded by the existing electron beam. This will ensure that the entire proton bunch self-modulates, as opposed to only the second half of the bunch, as in the current configuration [7]. The second milestone, in Run $2 b$, will be to demonstrate that the self-modulation process can be stabilized when the longitudinal wakefields reach their maximum amplitude, ensuring that the wakefields can be sustained for long timescales. This will require a sharp change in the plasma density, which will be possible in a dedicated plasma cell designed specifically for self-modulation [8]. Run $2 \mathrm{c}$ will require an additional plasma cell, to be installed downstream of the self-modulation cell, and a new electron beam, which will be injected between the two cells. This configuration will avoid interference between the self-modulation and acceleration processes, and it will use two known effects, beam loading the longitudinal wakefield and full blowout of plasma electrons, to preserve the quality of the electron beam [9].

The last goal, a scalable plasma source, can be achieved independently of the other two. The laser-ionization scheme currently used to generate the plasma cannot support long plasma cells, so two alternative schemes are currently being studied: one based on a low-frequency electromagnetic wave generated by RF antennas (Helicon) [10] and one based on a high-current arc in the plasma (Discharge) [11]

\section{Beyond Run 2: applications to high-energy particle physics}

A roadmap for particle physics applications of the AWAKE acceleration scheme has been laid out in a set of documents prepared for the European Particle Physics Strategy Update [12-14]. The roadmap takes into account our current knowledge, and proceeds from the more realistic goals to those that will be harder to achieve.

Fixed target experiments would be the first application of AWAKE. By extending the acceleration plasma cell from $10 \mathrm{~m}$ to $\mathrm{O}(100) \mathrm{m}$, energies of the order of $50 \mathrm{GeV}$ would be achievable. While the transverse beam quality would be too low for a collider, it would be sufficient for a fixed target experiment, and the electron flux would be several orders of magnitude higher than existing experiments at this energy, as shown in the tables of Figure 3. Such electron beams could significantly extend the reach of searches for rare particles such as dark photons, as well as probe strong-field QED electron/laser interactions, as shown in Figure 4.

As long as positron acceleration in wakefields remains a challenge, planning for an electronpositron machine is not realistic. However, the AWAKE scheme allows transformation of an existing hadron collider into an electron-proton or electron-ion collider, by using one of the proton beams to accelerate electrons to high energy, and colliding these electrons into the remaining proton/ion 
beam, as shown in Figure 5. Such electron-proton (or electron-ion) colliders would open a new energy regime for Deep Inelastic Scattering, significantly improving our current understanding of the proton/ion structure, and they would also extend the reach of direct searches for new particles such as leptoquarks, as shown in Figure 6.

To summarize, in Run 1 we have demonstrated that electrons can be accelerated in a plasma using a long proton bunch, taking advantage of the self-modulation process. In Run 2 we aim to control the beam quality and to demonstrate that we can build $\mathrm{O}(100) \mathrm{m}$ long plasma cells. Assuming Run 2 will be successful, we have started considering particle physics experiments which could be built using the AWAKE acceleration scheme. We explored a few examples for fixed target and electron-proton collisions, but of course there are many more opportunities to consider.

\begin{tabular}{lccc}
\hline $\begin{array}{c}\text { proton } \\
\text { energy }\end{array}$ & $\begin{array}{c}\text { plasma } \\
\text { length }\end{array}$ & $\begin{array}{c}\text { electron } \\
\text { energy }\end{array}$ & $\begin{array}{c}\text { electron } \\
\text { charge }\end{array}$ \\
\hline $400 \mathrm{GeV}$ & $50 \mathrm{~m}$ & $33 \mathrm{GeV}$ & $107 \mathrm{pC}$ \\
$400 \mathrm{GeV}$ & $100 \mathrm{~m}$ & $54 \mathrm{GeV}$ & $134 \mathrm{pC}$ \\
$450 \mathrm{GeV}$ & $130 \mathrm{~m}$ & $70 \mathrm{GeV}$ & $134 \mathrm{pC}$ \\
\hline
\end{tabular}

\begin{tabular}{lcc}
\hline Parameter & AWAKE-upgrade-type & HL-LHC-type \\
\hline Proton energy $E_{p}(\mathrm{GeV})$ & 400 & 450 \\
Number of protons per bunch $N_{p}$ & $3 \times 10^{11}$ & $2.3 \times 10^{11}$ \\
Longitudinal bunch size protons $\sigma_{z}(\mathrm{~cm})$ & 6 & 7.55 \\
Transverse bunch size protons $\sigma_{r}(\mu \mathrm{m})$ & 200 & 100 \\
Proton bunches per cycle $n_{p}$ & 8 & 320 \\
Cycle length $(\mathrm{s})$ & 6 & 20 \\
SPS supercycle length $(\mathrm{s})$ & 40 & 40 \\
\hline Electrons per cycle $N_{e}$ & $2 \times 10^{9}$ & $5 \times 10^{9}$ \\
\hline$\quad$ Number of electrons & $4.1 \times 10^{15}$ & $2 \times 10^{17}$ \\
on target per 12 weeks run & & \\
\hline
\end{tabular}

Figure 3: (Left) Electron energies achievable with different plasma lengths. (Right) Potential achievable number of electrons on target for different AWAKE-scheme fixed target configurations. For reference, the NA64 experiment at CERN expects $3 \times 10^{12}$ electrons during its entire lifetime [14].
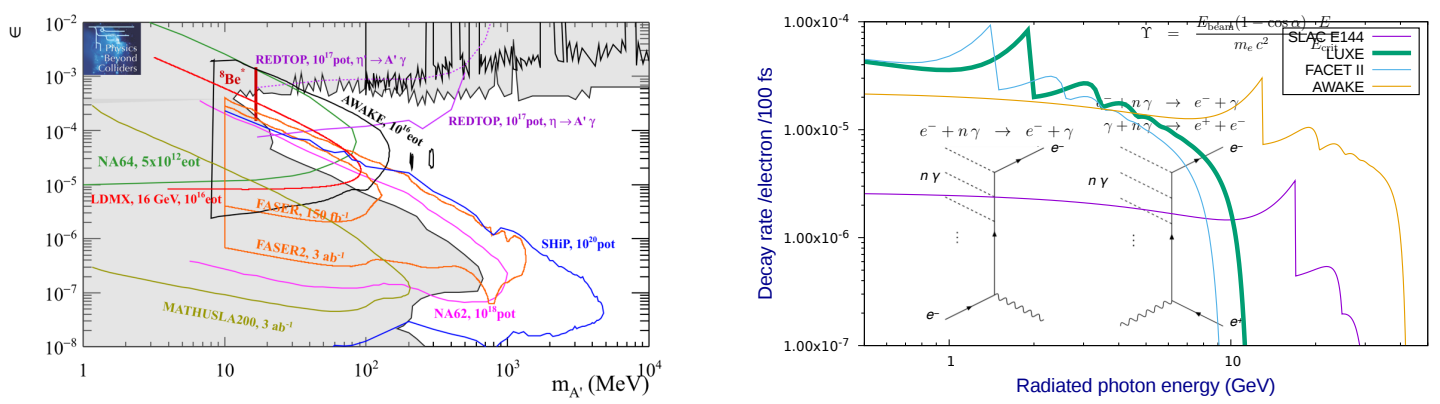

Figure 4: (Left) Dark photon sensitivity, as a function of coupling and mass, for an AWAKE-scheme fixed target experiment (black line), compared to existing limits (grey) and ongoing/planned experiments (colored lines). (Right) Strong-field QED sensitivity, in terms of electron/laser interaction rate as a function of radiated photon energy, for an AWAKE-scheme experiment, compared to other experiments $[13,15,16]$.

\section{References}

[1] P. Muggli, et al. (AWAKE Coll.), AWAKE readiness for the study of the seeded self-modulation of a $400 \mathrm{GeV}$ proton bunch, Plasma Phys. Control. Fusion 60 (2017) 014046 [1708.01087]. 

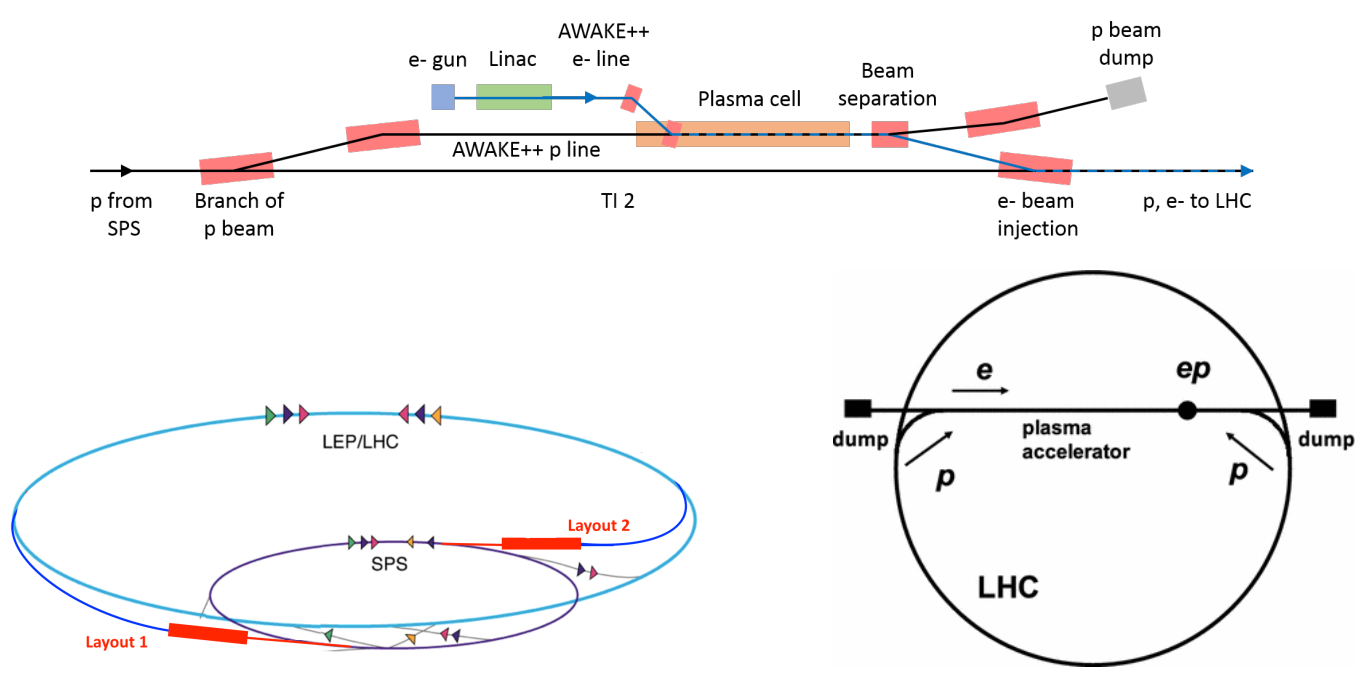

Figure 5: (Top) Schematic layout of an AWAKE-scheme electron accelerator for an electron/proton or electron/ion collider. Schematic layouts of the Plasma Electron Proton/Ion Collider (PEPIC), reaching a center-of-mass energy of $1.3 \mathrm{TeV}$ (left), and the Very High Energy electron-Proton collider (VHEeP), reaching $9 \mathrm{TeV}$ (right) $[17,18]$.

[2] M. Turner, et al. (AWAKE Coll.), Experimental observation of plasma wakefield growth driven by the seeded self-modulation of a proton bunch, Phys. Rev. Lett. 122 (2019) 054801 [1809.01191].

[3] E. Adli, et al. (AWAKE Coll.), Experimental observation of proton bunch modulation in a plasma, at varying plasma densities, Phys. Rev. Lett. 122 (2019) 054802 [1809. 04478].

[4] F. Batsch (on behalf of AWAKE Coll.), Setup and Characteristics of a Timing Reference Signal with sub-ps Accuracy for AWAKE, J. Phys. Conf. Ser. 1596 (2020) 012006 [1911.12201].

[5] E. Adli, et al. (AWAKE Coll.), Acceleration of electrons in the plasma wakefield of a proton bunch, Nature 561 (2018) 363 [1808.09759].

[6] P. Muggli (on behalf of AWAKE Coll.), Physics to plan AWAKE Run 2, J. Phys. Conf. Ser. 1596 (2020) 012008 [1911.07534].

[7] P. Muggli, et al., Seeding self-modulation of a long proton bunch with a short electron bunch, J. Phys. Conf. Ser. 1596 (2020) 012065 [2002 . 02189].

[8] A. Caldwell et al., Path to AWAKE: Evolution of the concept, Nucl. Instrum. Meth. A 829 (2016) 3 [1511.09032].

[9] V.K. Berglyd Olsen et al., Emittance preservation of an electron beam in a loaded quasilinear plasma wakefield, Phys. Rev. Accel. Beams 21 (2018) 011301 [1710.04858].

[10] B. Buttenschön et al., A high power, high density helicon discharge for the plasma wakefield accelerator experiment AWAKE, Plasma Physics and Controlled Fusion 60 (2018) 075005. 

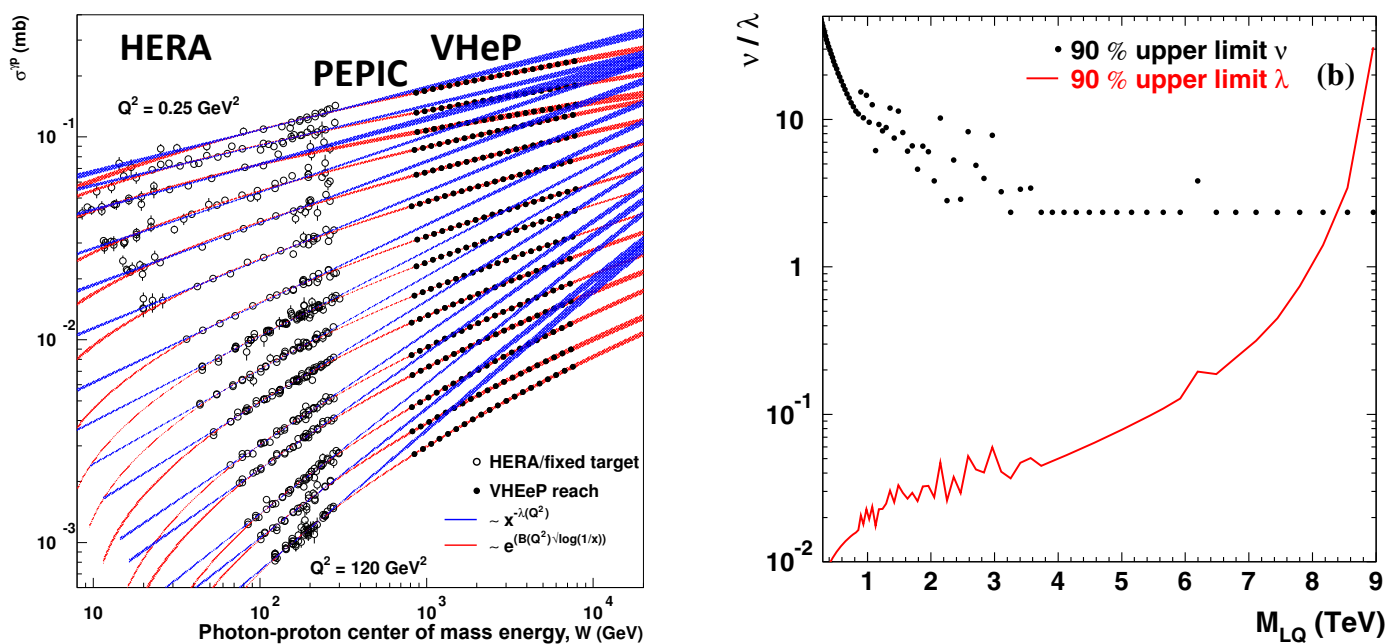

Figure 6: Physics reach of the Very High Energy electron-Proton collider (VHEeP). (Right) Measurement of photon-proton cross sections versus the center of mass energy, extending the reach of HERA. (Left) Upper limits on the number of events, $v$, and leptoquark coupling parameter, $\lambda$, versus mass of the leptoquark, $\mathrm{M}_{\mathrm{LQ}}[18]$.

[11] N.C. Lopes et al., Discharge plasma source, CALIFES Workshop 2016, https://indi.to/B6QhY.

[12] A. Caldwell et al., AWAKE: On the path to particle physics applications, 1812.08550.

[13] A. Caldwell et al., Particle physics applications of the AWAKE acceleration scheme, 1812.11164.

[14] E. Gschwendtner et al., AWAKE++: The AWAKE Acceleration Scheme for New Particle Physics Experiments at CERN, Tech. Rep. CERN-PBC-REPORT-2018-005, CERN, Geneva (Dec, 2018).

[15] J. Beacham et al., Physics Beyond Colliders at CERN: Beyond the Standard Model Working Group Report, J. Phys. G 47 (2020) 010501 [1901.09966].

[16] A. Hartin, Strong field QED in lepton colliders and electron/laser interactions, Int. J. Mod. Phys. A 33 (2018) 1830011 [1804.02934].

[17] G. Xia et al., Collider design issues based on proton-driven plasma wakefield acceleration, Nucl. Instrum. Meth. A 740 (2014) 173 [1404.6107].

[18] A. Caldwell et al., VHEeP: A very high energy electron-proton collider, Eur. Phys. J. C 76 (2016) 463 [1606.00783]. 